\title{
Autobiographical Script-Driven Imagery Has No Detectable Effect on Emotion Regulation in Healthy Individuals
}

\author{
Stephan Köhler ${ }^{\mathrm{a}}$ Veith Andreas Weilnhammer ${ }^{\mathrm{a}, \mathrm{c}}$ Henrik Walter ${ }^{\mathrm{a}}$ \\ Susanne Erk ${ }^{a}$ Philipp Sterzer ${ }^{a}$ b Anne Guhn ${ }^{a}$ \\ aDepartment of Psychiatry and Neurosciences, Charité - Universitätsmedizin Berlin, Corporate Member of Freie

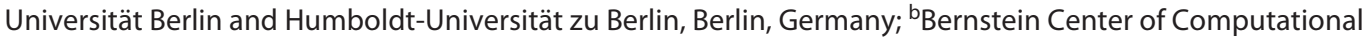 \\ Neuroscience, Berlin, Germany; 'Berlin Institute of Health, Charité-Universitätsmedizin Berlin and Max Delbrück \\ Center, Berlin, Germany
}

\section{Keywords}

Emotion regulation - Autobiographical script-driven imagery $\cdot$ Mood induction $\cdot$ Neuro correlates

\begin{abstract}
Introduction: Emotion regulation (ER), the ability to actively modulate one's own emotion reactions, likely depends on the individual's current emotional state. Here, we investigated whether negative emotions induced by an interpersonal autobiographic script affect the neuronal processes underlying ER. Methods: Twenty healthy participants were recruited and underwent functional magnetic resonance imaging (fMRI) during performance of distancing, a specific ER strategy, while viewing emotionally arousing pictures. Participants were instructed to either naturally experience ("permit" condition) or to actively downregulate ("regulate" condition) their emotional responses to the presented stimuli. Before each of the 4 runs in total, a neutral or negative autobiographical audio script was presented. The negative script comprised an emotionally negative event from childhood or adolescence that represented either emotional abuse or emotional neglect. The second event comprised an every-
\end{abstract}

day neutral situation. We aimed at identifying the neural correlates of ER and their modulation by script-driven imagery. Results: fMRI analyses testing for greater responses in the "regulate" than the "permit" condition replicated previously reported neural correlates of ER in the right dorsolateral prefrontal cortex and the right inferior parietal lobule. A significant ER effect was also observed in the left orbitofrontal cortex. In the amygdala, we found greater responses in the "permit" compared to the "regulate" condition. We did not observe a significant modulation of the ER effects in any of these regions by the negative emotional state induced by autobiographical scripts. Bayesian statistics confirmed the absence of such modulations by providing marginal evidence for null effects. Discussion: While we replicated previously reported neural correlates of $E R$, we found no evidence for an effect of mood induction with individualized autobiographical scripts on the neural processes underlying ER in healthy participants.

(c) 2021 The Author(s)

Published by S. Karger AG, Basel

Stephan Köhler and Veith Andreas Weilnhammer share first authorship. karger@karger.com www.karger.com/nps

Karger $\stackrel{\text { ' }}{5}$

BOPEN ACCESS
(C) 2021 The Author(s)

Published by S. Karger AG, Basel

This is an Open Access article licensed under the Creative Commons Attribution-NonCommercial-4.0 International License (CC BY-NC) (http://www.karger.com/Services/OpenAccessLicense), applicable to the online version of the article only. Usage and distribution for commercial purposes requires written permission.
Correspondence to:

Stephan Köhler, stephan.koehler@ charite.de 


\section{Introduction}

Emotions have a crucial role in everyday life and are of outstanding importance in interpersonal relationships. The ability to influence or regulate emotional responses, the so-called emotion regulation (ER), represents a critical component of healthy adaptation. According to Gross [1], ER describes “a person's ability to modulate his or her emotional state and expression that includes influencing which emotions people have, when they have these emotions, and how emotions are experienced and expressed." A wide range of mental disorders are characterized by impairments in emotion processing and, in particular, deficient ER [2-4]. ER may even act as a transdiagnostic treatment construct [5] across various psychiatric disorders. A range of ER strategies have been reported to be successful, including rumination, distraction, cognitive reappraisal, and suppression (Liu and Thompson [6]). "Distancing" is an ER strategy that involves simulating a new perspective to alter the psychological distance and emotional impact of a stimulus [7]. ER by distancing is effective in decreasing negative emotional experience and corresponding neural responses in the amygdala [8]. In general, the regulation of negative affect is associated with activity in frontal and parietal cortices [9]. More specifically, brain regions that show enhanced activity during distancing include the dorsolateral prefrontal cortex (DLPFC) and inferior parietal cortex and reduced activity in the amygdala $[7,10-12]$.

While the neural circuits involved in ER are thus fairly well established, less is known about the role of individual and contextual factors that may modulate ER, such as current emotional state and autobiographical context [6]. Here, we therefore investigated whether an individual mood induction using an interpersonal autobiographic script affects the neuronal processes underlying ER. We adopted an ER task that is well established and has been successfully used in previous studies [10-12]. In this paradigm, pictures with negative and neutral content are displayed following the instruction of either distancing or permit to the picture content. In the present study, we extended this paradigm with an autobiographical script to establish an individually specific mood induction and to test whether negative autobiographical memories change the neural responses associated with ER during viewing of negative affective pictures.

Based on the existing literature [7, 10-12], we focused our analyses on the DLPFC, inferior parietal cortex, and amygdala. We hypothesized that negative emotions induced by an autobiographical script would interfere with neural activity in these regions. In particular, based on previous work showing altered ER in depressed individuals [10], we expected to find enhanced amygdala and diminished DLPFC responses after script-driven negative mood induction. We here report the results in healthy participants, to investigate the combined mood induction (negative vs. neutral) and ER to evaluate the feasibility of this paradigm.

\section{Methods}

\section{Subjects}

The protocol was approved by the Ethics Committee of Charité - Universitätsmedizin Berlin and is in accordance with the Code of Ethics of the World Medical Association (Declaration of Helsinki). All subjects gave written informed consent.

In total, 20 healthy participants volunteered for the study. The mean age was 40.4 (SD: 13.2 years) and 55\% were females. The inclusion criterion was absence of a psychiatric disorder. Therefore, all participants were screened for major psychiatric axis I disorders using the Mini-International Neuropsychiatric Interview (M.I.N.I., [13]). The 21-item version of the Hamilton Depression Rating Scale was used to screen for current depressive symptoms (HAMD21, [14]) by trained psychologists. Moreover, participants completed the revised Beck Depression Inventory (BDI-II, [15]), the Toronto Alexithymia Scale (TAS-20, [16]), and the Emotion Regulation Questionnaire (ERQ, [17]). Exclusion criteria were any current or past psychiatric diagnosis or neurological brain diseases.

\section{Procedure}

Emotion Regulation Task

The software Presentation (Neurobehavioral Systems, Albany, CA, USA) was used for stimulus delivery. The ER task employed in the present experiment is well established and has been successfully used in previous studies [10-12]. The experimental paradigm was divided into 4 runs, each consisting of 15 trials. In each trial, participants were presented with a picture with either negative or neutral content.

Participants were instructed to either naturally experience all upcoming emotions ("permit" condition) or to cognitively downregulate their emotional responses by taking the position of a detached observer ("regulate" condition). Equal numbers of negative pictures were presented with the "permit" instruction and with the "regulate" instruction while neutral pictures were only presented with the "permit" instruction. Before fMRI imaging, participants were instructed carefully with example pictures and were trained with the distancing strategy. Distancing was chosen as an ER strategy as it can be explained and taught easily before the experiment. During the ER task in the fMRI scanner, subjects saw 60 pictures of negative or neutral content taken from the International Affective Picture System (IAPS, [18]). The IAPS pictures were taken from a previous study, which were matched across experimental conditions for complexity, content, color, and brightness as well as mean valence and arousal values (Dörfel et al. [11]). The experiment consisted of 3 conditions: "Permit-Neutral," "Permit-Negative," and "Regulate-Negative." For all conditions, subjects were 
instructed to look at the picture. In the conditions "Permit-Neutral" and "Permit-Negative," subjects were specifically instructed to "Look at the following picture directly and permit feeling your emotions," whereas in the condition "Regulate-Negative" they were told to regulate their emotions by using the distancing strategy. For the latter, participants were told to "look at the following picture directly but try to take the position of a noninvolved observer, thinking about the present picture in a neutral way." All subjects understood and could follow the instructions without difficulty. During scanning, each trial started with a cue word for $2 \mathrm{~s}$, reading either "permit" or "regulate," followed by an 8-s picture presentation. After each picture presentation, participants were asked to provide an arousal rating toward the pictures on a 9-point Likert-type scale ( $1=$ not unpleasant and $9=$ very unpleasant $)$. Trials concluded with a 14-s relaxation period with a fixation cross. Trials were presented in a pseudo-randomized order with a maximum of 2 consecutive trials of the same condition. The task was performed in 4 consecutive runs of $8 \mathrm{~min}$ each. Every run was preceded by an autobiographic audio script (neutral or negative, see below).

\section{Script-Driven Imagery}

Script-driven imagery procedures followed those of Pitman et al. [19], with 30-s script listening periods, adapted for fMRI [20]: participants were asked to recall 2 types of past experiences. The first type comprised an emotionally negative event from childhood or adolescence that represented either emotional abuse, for example, verbal assaults or humiliating behavior toward a child, or emotional neglect, for example, social exclusion or loneliness during childhood. The second type of experience comprised an everyday neutral situation, for example, taking a shower or brushing one's teeth [21]. Based on these individual reports, 2 scripts of 30-s duration were composed and recorded in a neutral female or male voice with regard to the participant's gender. Prior to each fMRI run, the neutral and negative scripts were presented in a randomized and block wise allocation, that is, 2 negative scripts followed by 2 neutral scripts or vice versa. The same script was played twice (e.g., 2 neutral and 2 negative scripts for each of the 4 runs). During the time of script presentation, a black screen was presented. Each subject was instructed to lie still and allow himself/herself to focus on the experience while listening to the script. Participants were further encouraged to remember all sensations that were associated with the events. As a manipulation check, participants had to fill out the Responses-to-ScriptDriven-Imagery Scale (RSDI) at the end of the experiment (Hopper et al. [22]). The RSDI is an 11-item instrument for assessing severities of state re-experiencing ( 4 items), avoidance ( 3 items), and dissociative symptoms ( 4 items) provoked by script-driven trauma imagery, with items rated from 0 (not at all) to 6 (a great deal).

\section{Behavioral Data Analysis}

Behavioral data analysis was conducted with SPSS Statistics Version 25 (SPSS Inc., an IBM Company). For differences between negative and neutral audio scripts in the RSDI ratings, we used 1-way ANOVA and the Kruskal-Wallis test for non-normally distributed data, respectively. To analyze the differences of negative feelings between previously regulated and nonregulated negative pictures in the post hoc ratings of negative emotions, we used repeated-measures ANOVA (rANOVA).

Script-Driven Imagery and Emotion

Regulation

\section{fMRI Methods}

Acquisition and Preprocessing

We acquired blood-oxygen level-dependent (BOLD) images using $\mathrm{T}^{*}$-weighted gradient-echo echo-planar imaging (FOV 192,33 slices, TR 2,000 ms, TE $30 \mathrm{~ms}$, flip angle $78^{\circ}$, voxel size $3 \times$ $3 \times 3 \mathrm{~mm}$, and interslice gap $10 \%$ ) on a 3T MRI scanner (Tim Trio; Siemens) at the Berlin Centro of Advanced Neuroimaging (BCAN). For each of the 4 experimental runs, we recorded 228 volumes. Furthermore, we collected anatomical images using a T1-weighted MPRAGE sequence (FOV 256, 160 slices, TR 1,900 ms, TE 2.52 $\mathrm{ms}$, flip angle $9^{\circ}$, and voxel size $1 \times 1 \times 1 \mathrm{~mm}$ ).

Image preprocessing was carried out using statistical parametric mapping as implemented in SPM12 (http://www.fil.ion.ucl. ac.uk/spm/software/spm12/) for image preprocessing. Specifically, we applied standard realignment, coregistration, normalization to MNI stereotactic space with unified segmentation, and spatial smoothing using an 8-mm full-width at half maximum isotropic Gaussian kernel.

\section{fMRI Data Analysis}

In this work, we aimed at identifying the neural correlates of processes involved in ER in healthy controls and their modulation by a script-driven imagery. To this end, we used a mixed eventrelated and block design. As regressors-of-interest, we defined all combinations of the specific instruction ("regulate" vs. "permit") and the emotional content of the picture ("negative" vs. "neutral"). Since the neutral pictures were only presented together with the "permit" condition, we extracted 3 distinct regressors ("Permitneutral," "Permit-negative," and "Regulate-negative"), which were modeled as box-car regressors with onsets aligned to the onsets of the visual stimuli and a duration of $8 \mathrm{~s}$. For these regressors, we defined additional parametric regressors using the trial-wise emotional rating ("Permit-neutral $\times$ Rating," "Permit-negative $\times$ Rating," and "Regulate-negative $\times$ Rating"). Furthermore, we defined regressors-of-no-interest comprising the presentation of the ER instruction screen ("Instruction") and rating screen ("Rating"), both represented by box-car functions.

We convolved all regressors with the canonical hemodynamic response function implemented in SPM12. In addition, the design matrix included 6 rigid-body realignment parameters as nuisance covariates. After high-pass filtering at $1 / 128 \mathrm{~Hz}$, we estimated single-subject statistical parametric maps. We calculated contrast images for all regressors versus baseline as well as for the following combinations of factors: "Permit-negative" + "Regulate-negative" versus "Permit-neutral" and "Regulate-negative" versus "Permitnegative." All contrast images were calculated separately for runs preceded by "neutral" and "negative" autobiographical scripts and submitted to paired $t$ tests on the second level. This allowed us to assess neural responses irrespective of the autobiographical scripts as well as differences between runs preceded by "negative" as compared to "neutral" autobiographical scripts. We performed anatomic labeling of second-level cluster peaks using the SPM Anatomy Toolbox Version 1.7b [23].

In the first step, we tested for general effects of affective versus neutral pictures ("Permit-negative" + "Regulate-negative" vs. "Permit-neutral") and for neural correlates of ER ("Regulate-negative" vs. "Permit-negative") at the group level. We performed analyses across the whole brain and within regions-of-interest (ROIs) that were defined for the whole group. These group ROIs comprised regions reported by previous studies investigating ER with the same 
paradigm [7, 10-12], that is, the right DLPFC and the right inferior parietal lobule (IPL) for "Regulate-negative" > "Permit-negative" and the amygdala for the reverse contrast. For the DLPFC and IPL, we defined spherical group ROIs around the previously reported peak coordinates for the contrast "Regulate-negative" > "Permitnegative" (DLPFC: [39 24 42]) and inferior parietal lobule (IPL: [51 $-6342]$, see [11]). To define the amygdala group ROI, we mapped the second-level contrast for "Permit-negative" + "Regulate-negative" versus "Permit-Neutral" at a threshold of $p<0.005$, uncorrected. Please note that this contrast is orthogonal to the contrast "Regulate-negative" versus "Permit-negative" and to the contrasts testing for autobiographical script effects (see below). To only select voxels within the anatomical boundaries of the amygdala, we intersected the resulting contrast image with an anatomical mask for the left amygdala as provided by the Neuromorphometrics-Toolbox within SPM12. To determine statistical significance, we used family-wise error (FWE) correction across the whole brain or smallvolume correction within the ROIs.

In the second step, we tested for modulations of ER effects by the negative emotional state induced by preceding autobiographical scripts. In an exploratory whole-brain analysis, we used voxel-wise rANOVA to test for interaction of the within-subject factors "Regulation Instruction" (i.e., "permit" vs. "regulate") and "Autobiographical Script" (i.e., "negative" vs. "neutral script"). Effects were considered significant at $p<0.05$, FWE corrected across the whole brain.

To maximize sensitivity for a potential interaction effect in our hypothesis-driven ROI analyses, we defined individual subjectspecific ROIs within the group ROIs described above, that is, for the DLPFC, the IPL, and the amygdala, as well as for another region that showed a significant ER effect in our whole-brain analysis, the left orbitofrontal cortex (OFC, middle orbital gyrus [-42 47 -1 ]; see fMRI results - whole-brain analyses).

Individual ROIs were defined as spherical volumes of 5-mm radius around the peak voxel of each individual participants' contrast "Regulate-negative" versus "Permit-negative" within each group ROI. Please note again that this contrast is orthogonal to the contrast testing for effects of the preceding autobiographical script on ER. We derived parameter estimates using the MarsBaR Toolbox 0.42 (http://marsbar.sourceforge.net) and averaged across all voxels within the individual ROIs. Run-wise averaged parameter estimates for the regressors "Regulate-negative" and "Permit-negative" were submitted to rANOVA with the 2 within-subject factors "Regulation Instruction" and "Autobiographical Script" (see above).

Effects of ROI analyses were considered significant at $p<0.05$, Bonferroni-corrected for the number of ROIs. In addition, we computed Bayes Factors (BF10) for both main effects and the between-factor interaction using "anovaBF" (Jeffreys prior for fixed effects at an $r$ scale value of one-half) from the "BayesFactor" $\mathrm{R}$ package. We interpret BF $10>3$ as evidence confirming the alternative hypothesis. In turn, $\mathrm{BF} 10<0.3$ is taken as evidence for the null hypothesis [24].

\section{Results}

\section{Behavioral Data}

None of the participants showed relevant depressive symptoms (HAMD-21; mean: $1.4 \pm 0.40$; BDI, mean: 2.1 \pm 0.47 ) or had a psychiatric disorder according to the M.I.N.I. The mean of the TAS was $54.8 \pm 1.63$ indicating that participants had no alexithymia. Values regarding ER (ERQ_reappraisal; mean: $4.31 \pm 0.15$; ERQ_suppression; mean: $3.22 \pm 0.12$ ) were also comparable to values of other studies with healthy participants [17], indicating that participants did not have self-reported ER problems. All participants reported a significantly higher re-experiencing sum score (including items of unpleasant feelings, to feel stressed, and the feeling of re-experiencing the situation) after the negative audio script ( $m=9.05 \pm 0.98$ ) compared to the neutral audio script $(m=3.42 \pm 1.01$; $T=8.8, p<0.05$ ), indicating the affective involvement.

Furthermore, we obtained arousal ratings in the 2 script-driven imagery conditions for neutral pictures (neutral autobiographical script: $1.63 \pm 0.17$; negative autobiographical script: $2.15 \pm 0.33$ ), regulated negative pictures (neutral autobiographical script: $3.95 \pm 0.30$; negative autobiographical script: $3.95 \pm 0.36$ ), and unregulated negative pictures (neutral autobiographical script: $5.60 \pm 0.42$; negative autobiographical script: $6.03 \pm 0.42$ ). For the factors "valence" (neutral vs. negative) and "autobiographical script" (neutral vs. negative), rANOVA showed a main effect of emotional valence $(F=93.35, p<0.001)$, no main effect of autobiographical script $(F=1.94, p=0.18)$, and no between-factor interaction $(F=0.48, p=0.50)$. For the factors "regulation" (regulate vs. permit) and "autobiographical script" (neutral vs. negative), rANOVA yielded a main effect of "regulation" $(F=24.61, p<0.001)$, no main effect of autobiographical script $(F=0.35, p=0.57)$, and no between-factor interaction $(F=0.47, p=0.50)$.

\section{fMRI Results}

\section{Effects of Emotionally Arousing Pictures}

We first mapped the activity corresponding to negative versus neutral emotional content irrespective of the regulation instruction and autobiographical script. Similar to previous reports, whole-brain analysis using this contrast ("Permit-negative" + "Regulate-negative" vs. "Permitneutral") revealed clusters in the bilateral fusiform gyrus (left: $[-39-55-13], T=7.20$; right: $[-39-61-16], T=$ 6.70), left inferior occipital gyrus ([-36 -76 -10], $T=$ 6.58 ), and right inferior temporal gyrus ([48 $-70-7], T=$ $6.39)$ at $p<0.05$ (FWE corrected). Comparing this contrast between runs preceded by negative versus neutral autobiographical scripts did not yield any significant results.

\section{Effects of Emotion Regulation}

Whole-brain analysis using the contrast "Regulatenegative" versus "Permit-negative" revealed clusters in 
the right IPL ([51 -6342$], T=6.68)$ and left OFC ([-42 $47-1], T=6.51$ ) at $p<0.05$, FWE corrected (Fig. 1). Furthermore, literature-based ROI analysis additionally revealed significant activation in the right DLPFC ([39 24 $42], T=5.00$; see [11]).

\section{Effects of Mood Induction}

In our individual ROI-based analyses (see Fig. 2), we tested for differences in parameter estimates between the regressors "Regulate-negative" and "Permit-negative" separately for runs preceded by "neutral" as opposed to "negative" autobiographical scripts. To this end, we performed rANOVAs with 2 within-subject factors: "Regulation Instruction" (i.e., "Permit" vs. "Regulate") and "Autobiographical Script" (i.e., "negative” vs. "neutral”).

In the amygdala, we observed a significant main effect of "Regulation Instruction" (F [57] $=5.77, p=0.02$, BF_10 = 3.29), with lower parameter estimates in the "Regulate" condition. BOLD activity in this region did not show a main effect of "Autobiographical Script" (F [57] $=2.27$, $p=0.14, \mathrm{BF} \_10=0.73$ ) or a between-factor interaction, the Bayes factor for the latter suggesting marginal evidence for a null effect $\left(\mathrm{F}[57]=0.03, p=0.86, \mathrm{BF} \_10=0.37\right)$.

For the DLPFC ROI, we found a main effect for the factor "Regulation Instruction" (F [57] $=6.24, p=0.02$, BF_10 $=3.16)$ with greater parameter estimates for "Regulate-negative" as compared to "Permit-negative." This main effect was expected because the ROI was defined using this contrast. We also observed a marginal main effect for the factor "Autobiographical Script" (F [57] = $4.23, p=0.04, \mathrm{BF} \_10=1.23$ ), with reduced parameter estimates following negative autobiographical scripts. We did not find any interaction between "Regulation Instruction" by "Autobiographical Script," again with marginal evidence for a null effect $\left(\mathrm{F}[57]=0.31, p=0.58, \mathrm{BF} \_10=\right.$ $0.33)$.

Likewise, for the IPL ROI, we observed the expected main effect of "Regulation Instruction" (F [57] = 13.49, $p=5.32 \times 10^{-4}$, BF_10 $\left.=64.34\right)$ and a marginal effect of "Autobiographical Script" (F [57] $=4.3, p=0.04$, BF_10 = 1.54), but no between-factor interaction, with marginal evidence for a null effect $\left(\mathrm{F}\right.$ [57] $=0.15, p=0.7, \mathrm{BF} \_10=$ $0.31)$. For the OFC ROI, we observed the trend for a main effect of "Regulation Instruction" (F [57] $=3.39, p=0.07$, BF_10 = 1.04). In OFC, parameter estimates were significantly lower following negative autobiographical scripts, a main effect of "Autobiographical Script," (F [57] = 8.01, $p=6.4 \times 10^{-3}, \mathrm{BF} \_10=7.38$ ). Again, we did not find a significant between-factor interaction, with marginal evidence for a null effect $\left(\mathrm{F}[57]=0.08, p=0.78, \mathrm{BF} \_10=\right.$

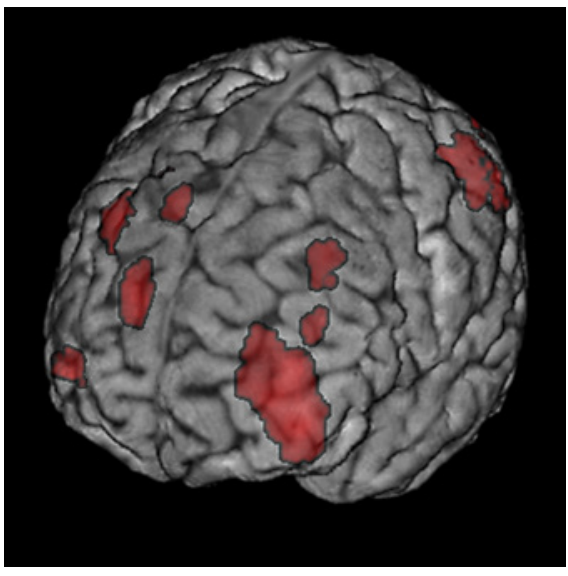

Fig. 1. "Regulate-negative" versus "Permit-negative": this contrast revealed significant clusters (FWE corrected across the whole brain) in the right inferior parietal lobule ([51 -63 42], $T=6.68)$ and left middle orbital gyrus ([-42 $47-1], T=6.51)$. Region-ofinterest analysis also showed a significant effect of ER in the right dorsolateral prefrontal cortex ([39 24 42],$T=5.00$ ). Displayed at $p<0.001$, uncorrected.

0.32 ). Additional exploratory whole-brain analyses for effects of mood induction on ER revealed no significant effect for the interaction of the factors "Regulation Instruction" and "Autobiographical Script" at our predefined significance threshold of $p<0.05$, FWE corrected.

\section{Discussion}

The present study investigated whether individualized mood induction using interpersonal autobiographical scripts modulates the instructed regulation of negative emotions and its neural correlates. fMRI analyses showed greater responses in the "regulate" than the "permit" condition in the right DLPFC and the right inferior parietal lobule. A significant ER effect was also observed in the left orbitofrontal cortex. In the amygdala, we found greater responses in the "permit" compared to the "regulate" condition. We did not observe a significant modulation of the ER effects in any of these regions by the negative emotional state induced by autobiographical scripts. While we thus replicated previously reported neural correlates of ER in frontoparietal regions and the amygdala [10-12], we found no evidence for an effect of mood induction with individualized autobiographical scripts on the neural processes underlying ER.

There are a number of possible explanations for the absence of a significant effect of mood induction on the neu- 


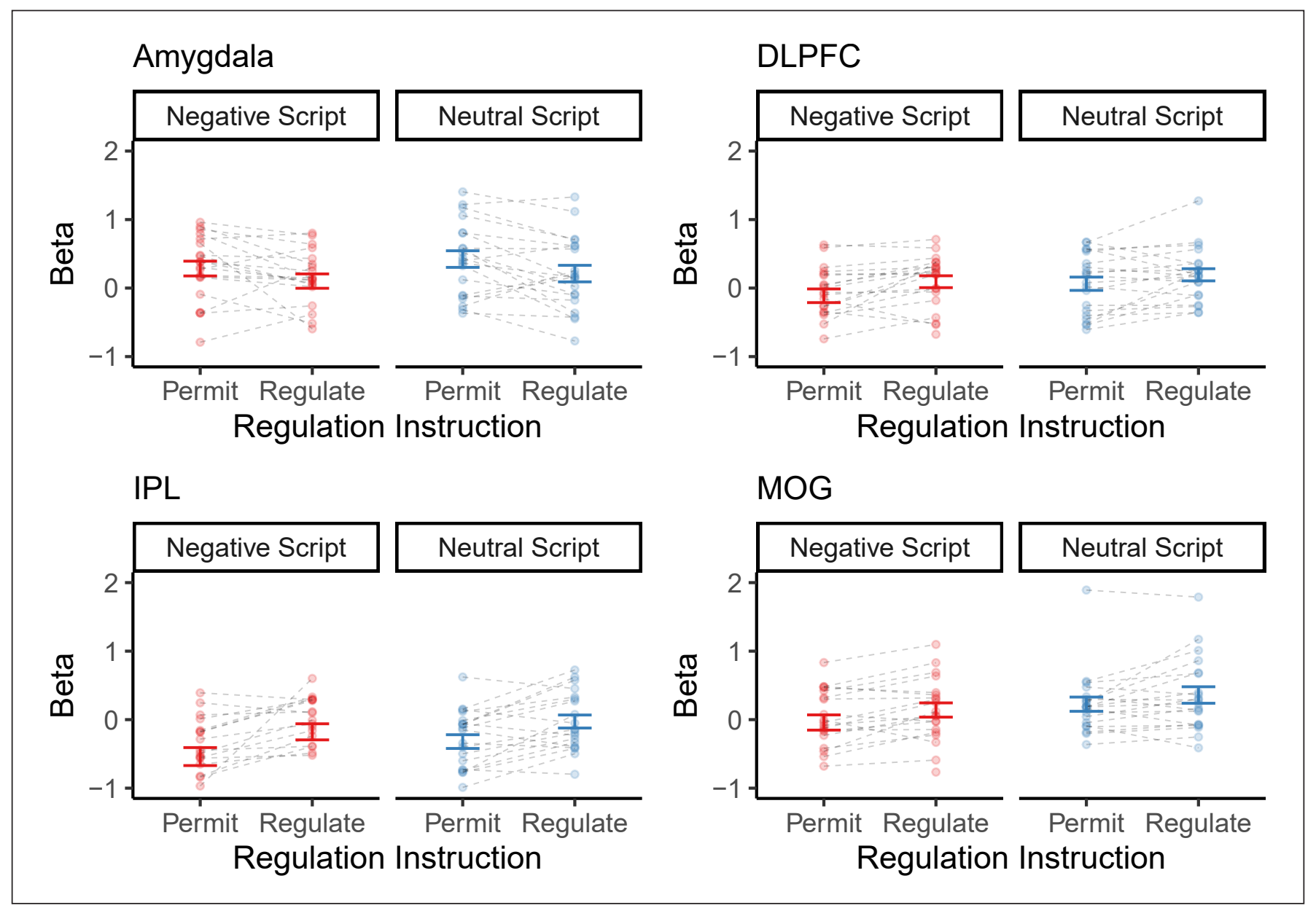

Fig. 2. ROI-based analyses. In the amygdala, the BOLD signal was significantly greater during the Permit compared to the Regulate condition, while script-driven imagery had no detectable effect. In the DLPFC, the BOLD signal was significantly greater during the Regulate compared to the Permit condition. Likewise, for the IPL, we observed a BOLD signal, which was greater during the Regulate

ral correlates of ER in our study: first, the sample size of our study may have been too small. However, Bayesian statistics suggested that modulatory effects of mood induction on BOLD activity were indeed absent in our data, rather than being due to insufficient statistical power. Second, the autobiographical scripts may not have been effective in triggering negative mood. However, we found a significant difference in self-rated arousal by the negative autobiographical script compared to the neutral script (rated by the re-experiencing subscale of the RSDI). Thus, the autobiographical script indeed induced (negative) emotions, in line with the results of previous studies using autobiographical scripts in a comparable way [22, 25, 26]. Still, mood induction in our study may not have been strong

compared to the Permit condition. For the MOG ROI, we observed a trend for a stronger BOLD signal during the Regulate compared to the Permit condition. ROI, region-of-interest; DLPFC, dorsolateral prefrontal cortex; IPL, inferior parietal lobule; MOG, middle orbital gyrus; BOLD, blood-oxygen level-dependent.

enough to modulate ER. Therefore, not pretesting the scripts for their emotional activity is a limitation of our study. Third, the mood induction procedure may have produced only a short-lived emotional state insufficient to affect ER throughout the subsequent fMRI run. Fourth, it is conceivable that the healthy participants tested in our current study may have been able to maintain their ER abilities despite successful negative mood induction. In contrast, individuals with increased susceptibility to negative autobiographical memories may show an effect of script-based negative mood induction on ER. In other words, our experimental paradigm may not have been sensitive enough to detect effects of mood induction on ER, at least in healthy participants. Finally, ER may be generally immune to neg- 
ative mood induction. One way to distinguish between the latter 2 explanations in future studies may be the inclusion of groups of individuals whose psychopathology suggests a relationship between negative autobiographical memories and deficits in ER, such as patients with chronic depression $[21,27]$. With regard to the effect of the scripts on the behavioral and neural level, in future studies, the autobiographical scripts could be pretested to evaluate subjective and physiological effects (e.g., valence, arousal, and heart rate). In a recent review, Mikulincer and Shaver [28] found evidence that individual differences in attachment orientation are reflected in cognitive, behavioral, and neural patterns of ER. If these patterns can be activated by negative autobiographical memories that relate to attachment orientation in an experimental design, this could help to characterize psychiatric disorders much more specifically. Indeed, emotional autobiographical stimuli were found to differentially impact the ability of ER in depressive patients [29]. Moreover, patients with depression demonstrated elevated amygdala activity and greater amygdala-hippocampal connectivity after listening to a negative autobiographical script followed by a cognitive ER task (to regulate the autobiographical script) compared to healthy controls [30]. This could be important for the development of therapeutic interventions that can counteract anxious hyperactivation of distress-related feelings and thoughts, which are activated in specific (interpersonal) situations. Based on these considerations, the here presented paradigm may be a useful tool to study ER in selected groups of psychiatric patients, for example, those with chronic depression.

The idea of mood induction by an interpersonal autobiographical script that alters processes like ER emphasizes the meaning of social relationships and social functioning especially for the development of mental disorders. There are different recent approaches to investigate the neural mechanism of social encounters, for example, "the secondperson neuroscience" [31]. Especially the latter one offers the chance to investigate the effects of current social interactions on specific processes in human behavior, which is of relevance also for future neuroimaging studies [32]. With regard to our null findings, this approach could offer a more stimulating (e.g., mood induction) and reliable (examination of interaction) experimental environment.

\section{Statement of Ethics}

The protocol was approved by the Ethics Committee of Charité Universitätsmedizin Berlin (EA1/324/15) and is in accordance with the Code of Ethics of the World Medical Association (Declaration of Helsinki). All subjects gave written informed consent.

\section{Conflict of Interest Statement}

The authors declare that the research was conducted in the absence of any commercial or financial relationships that could be construed as a potential conflict of interest.

\section{Funding Sources}

This work was funded by the German Research Foundation (DFG, KO 5231/2-1) and by the Else Kröner-Fresenius-Stiftung (Project "Psychopathologie und Neurobiologie chronisch depressiver Erkrankungen,” nb. 2018_A142, Leader: Stephan Köhler).

\section{Author Contributions}

S.K. contributed to conceptualization, data collection, data analyses, and writing the original draft. . V.A.W. carried out the functional imaging analyses. A.G. contributed to quantitative data analysis and editing the draft. H.W. contributed to qualitative data analysis, conceptualization, and editing the draft. S.E. contributed to editing the draft and paradigm design. P.S. contributed to conceptualization, supervision of data analyses, and editing the draft. S.K. contributed to conceptualization, funding acquisition, and editing the draft.

\section{Data Availability Statement}

The data that support the findings of this study are not publicly available because they contain information that could compromise the privacy of research participants but are available from the corresponding author S.K. upon reasonable request.

References

Neuropsychobiology 2022;81:141-148 DOI: $10.1159 / 000518996$ $217-32$.
1 Gross JJ. The emerging field of emotion regulation: an integrative review. Rev Gen Psychol. 1998;2(3):271-99.

2 Pico-Perez M, Radua J, Steward T, Menchon JM, Soriano-Mas C. Emotion regulation in mood and anxiety disorders: a meta-analysis of fMRI cognitive reappraisal studies. Prog Neuropsychopharmacol Biol Psychiatry. 2017 Oct 3;79(Pt B):96-104.

3 Visted E, Vøllestad J, Nielsen MB, Schanche E. Emotion regulation in current and remitted depression: a systematic review and metaanalysis. Front Psychol. 2018;9:756.

4 Daros AR, Williams GE. A meta-analysis and systematic review of emotion-regulation strategies in borderline personality disorder. Harv Rev Psychiatry. 2019 Jul/Aug;27(4):

5 Sloan E, Hall K, Moulding R, Bryce S, Mildred $\mathrm{H}$, Staiger PK. Emotion regulation as a transdiagnostic treatment construct across anxiety, depression, substance, eating and borderline personality disorders: a systematic review. Clin Psychol Rev. 2017 Nov;57:141-63. 
6 Liu DY, Thompson RJ. Selection and implementation of emotion regulation strategies in major depressive disorder: an integrative review. Clin Psychol Rev. 2017 Nov;57:183-94.

7 Koenigsberg HW, Fan J, Ochsner KN, Liu X, Guise K, Pizzarello S, et al. Neural correlates of using distancing to regulate emotional responses to social situations. Neuropsychologia. 2010 May;48(6):1813-22.

8 Powers JP, LaBar KS. Regulating emotion through distancing: a taxonomy, neurocognitive model, and supporting meta-analysis. Neurosci Biobehav Rev. 2019 Jan;96:155-73.

9 Ochsner KN, Gross JJ. Cognitive emotion regulation: insights from social cognitive and affective neuroscience. Curr Dir Psychol Sci. 2008 Apr;17(2):153-8.

10 Erk S, Mikschl A, Stier S, Ciaramidaro A, Gapp V, Weber B, et al. Acute and sustained effects of cognitive emotion regulation in major depression. J Neurosci. 2010 Nov 24; 30(47):15726-34.

11 Dörfel D, Lamke JP, Hummel F, Wagner U, Erk S, Walter H. Common and differential neural networks of emotion regulation by detachment, reinterpretation, distraction, and expressive suppression: a comparative fMRI investigation. Neuroimage. 2014 Nov 1;101: 298-309.

12 Lamke JP, Daniels JK, Dörfel D, Gaebler M, Abdel Rahman R, Hummel F, et al. The impact of stimulus valence and emotion regulation on sustained brain activation: task-rest switching in emotion. PLoS One. 2014;9(3): e93098.

13 Sheehan DV, Lecrubier Y, Sheehan KH, Amorim P, Janavs J, Weiller E, et al. The miniinternational neuropsychiatric interview (M.I.N.I.): the development and validation of a structured diagnostic psychiatric interview for DSM-IV and ICD-10. J Clin Psychiatry. 1998;59 Suppl 20:22-33; quiz 34-57.

14 Hamilton M. A rating scale for depression. J Neurol Neurosurg Psychiatry. 1960 Feb;23: $56-62$.
15 Beck AT, Ward CH, Mendelson M, Mock J, Erbaugh J. An inventory for measuring depression. Arch Gen Psychiatry. 1961 Jun;4: 561-71.

16 Bach M, Bach D, de Zwaan M, Serim M, Böhmer F. Validierung der deutschen Version der 20-Item Toronto-Alexithymie-Skala bei Normalpersonen und psychiatrischen patienten. Psychother Psychosom Med Psychol. 1996; 46(1):23-8.

17 Abler B, Kessler H. Emotion regulation questionnaire: Eine deutschsprachige Fassung des ERQ von Gross und John. Diagnostica. 2009; 55(3):144-52.

18 Lang PJ, Bradley MM, Cuthbert BN. International affective picture system (IAPS): affective ratings of pictures and instruction manual. Technical report A-8. Gainesville, FL: University of Florida; 2008.

19 Pitman RK, Orr SP, Forgue DF, de Jong JB, Claiborn JM. Psychophysiologic assessment of posttraumatic stress disorder imagery in Vietnam combat veterans. Arch Gen Psychiatry. 1987 Nov;44(11):970-5.

20 Lanius RA, Williamson PC, Boksman K, Densmore M, Gupta M, Neufeld RW, et al. Brain activation during script-driven imagery induced dissociative responses in PTSD: a functional magnetic resonance imaging investigation. Biol Psychiatry. 2002 Aug 15; 52(4):305-11.

21 Guhn A, Sterzer P, Haack FH, Köhler S. Affective and cognitive reactivity to mood induction in chronic depression. J Affect Disord. 2018 Jan 3;229:275-81.

22 Hopper JW, Frewen PA, Sack M, Lanius RA, van der Kolk BA. The responses to scriptdriven imagery scale (RSDI): assessment of state posttraumatic symptoms for psychobiological and treatment research. J Psychopathol Behav Assess. 2007;29(4):249-68.

23 Eickhoff SB, Stephan KE, Mohlberg H, Grefkes C, Fink GR, Amunts K, et al. A new SPM toolbox for combining probabilistic cytoarchitectonic maps and functional imaging data. Neuroimage. 2005 May 1;25(4):132535.
24 Wetzels R, Wagenmakers E-J. A default Bayesian hypothesis test for correlations and partial correlations. Psychon Bull Rev. 2012; 19(6): 1057-64. https: //doi.org/10.3758/ s13423-012-0295-x.

25 Lanius RA, Frewen PA, Girotti M, Neufeld RW, Stevens TK, Densmore M. Neural correlates of trauma script-imagery in posttraumatic stress disorder with and without comorbid major depression: a functional MRI investigation. Psychiatry Res. 2007 May 15; 155(1):45-56.

26 Frijling JL, van Zuiden M, Koch SB, Nawijn L, Veltman DJ, Olff M. Intranasal oxytocin affects amygdala functional connectivity after trauma script-driven imagery in distressed recently trauma-exposed individuals. Neuropsychopharmacology. 2016 Apr;41(5):128696.

27 Guhn A, Steinacher B, Merkl A, Sterzer P, Köhler S. Negative mood induction: affective reactivity in recurrent, but not persistent depression. PLoS One. 2019;14(1):e0208616.

28 Mikulincer M, Shaver PR. Attachment orientations and emotion regulation. Curr Opin Psychol. 2019 Feb;25:6-10.

29 Doré BP, Rodrik O, Boccagno C, Hubbard A, Weber J, Stanley B, et al. Negative autobiographical memory in depression reflects elevated amygdala-hippocampal reactivity and hippocampally associated emotion regulation. Biol Psychiatry Cogn Neurosci Neuroimaging. 2018 Apr;3(4):358-66.

30 Dore BP, Rodrik O, Boccagno C, Hubbard A, Weber J, Stanley B, et al. Negative autobiographical memory in depression reflects elevated amygdala-hippocampal reactivity and hippocampally associated emotion regulation. Biol Psychiatry Cogn Neurosci Neuroimaging. 2018 Apr;3(4):358-66.

31 Schilbach L, Timmermans B, Reddy V, Costall A, Bente G, Schlicht T, et al. Toward a second-person neuroscience. Behav Brain Sci. 2013;36(4):393-414.

32 Schilbach L. Towards a second-person neuropsychiatry. Philos Trans R Soc Lond B Biol Sci. 2016;371(1686):20150081. 